\title{
Performance prediction of pump and pumping system based on combination of numerical simulation and non-full passage model test
}

\author{
Honggeng Zhu' ${ }^{1} \cdot \mathrm{Ge} \mathrm{Bo}^{2} \cdot{\text { Yuanbin } \mathrm{Zhou}^{3} \cdot \text { Rentian Zhang }}^{2} \cdot$ Jilin Cheng $^{1}$
}

Received: 11 May 2018 / Accepted: 18 August 2019 / Published online: 27 August 2019

(c) The Author(s) 2019

\begin{abstract}
The design scheme of open intake will often increase the shaft length and the height of wet-pit pump house when the range of intake water level varies relatively large. And if the pump house is far away from the discharge bay, a longer discharge pipe may be inevitable, and the arrangement of full passage model pumping system may exceed the allowed size of a specific test bench. Aiming at a long-shaft vertical pumping system with open intake design, larger water level variation and longer discharge pipe, a methodology of performance prediction for the pump and pumping system was proposed by combining numerical simulation and non-full passage model test because of less experiment funds, shorter research periods and restricted test bench. Through numerical simulation, the hydraulic losses and drag coefficients of sump and discharge pipe were calculated and followed by the experiment design and model test of non-full passage pumping system. Based on the calibration accuracy of measurement instruments and test results, the experimental uncertainties were analyzed. Through combination of numerical simulation and non-full passage model test, the prediction of model pump and pumping system performance was realized. The performance of prototype pump and pumping system was obtained by applying the similarity law of pump. When operated under the designed head of $13.59 \mathrm{~m}$, the flow rate and efficiency of the prototype pumping system reached $11.86 \mathrm{~m}^{3} / \mathrm{s}$ and $86.56 \%$, respectively, exceeding the requirements stipulated in the bidding documents and showing that it possessed greater pumping capacity and higher efficiency. The methodology proposed in this paper can be referenced and applied to the engineering design of similar pump stations.
\end{abstract}

Keywords Pump and pumping system $\cdot$ Numerical simulation $\cdot$ Model test $\cdot$ Performance prediction $\cdot$ Non-full passage $\cdot$ Similarity law

\section{Introduction}

A pumping system consists of an intake structure, a pump and an outflow structure. Model pumping system test is generally required in China for newly built or technological transformation of large and medium pump stations, to

Technical Editor: Jader Barbosa Jr., Ph.D.

Honggeng Zhu

hgzhu@163.com

1 College of Hydraulic Science and Engineering, Yangzhou University, Yangzhou 225001, China

2 Jiangsu Surveying and Design Institute of Water Resources Co. Ltd., Yangzhou 225127, China

3 Luoyun Water Conservancy Project Management Office of Jiangsu Province, Shuqian 223800, China verify the rationality of pump selection and pump station design, to check the pump performance committed by pump manufactures and to conduct revised design if necessary, etc. In order to reduce the investment in civil engineering, open sump and wet-pit pump house is often selected as the intake structure of a pump station, which possesses many advantages such as simple structure and convenient construction. However, the shaft length and height of pump house will be increased accordingly in occasion of larger variation of intake water level [1,2]. To carry out model test of hydraulic and cavitation performance for such a pumping system will require more spacious test bench; otherwise, the requirement of larger variation of intake water level and higher pumping sets cannot be satisfied. Moreover, some water-delivering pump stations have longer discharge pipe. All of these factors make it hardly impossible to carry out model test of full passage pumping system on ordinary test benches mainly 
due to the restriction of allowed maximum length and height $[3,4]$.

CFD analysis previously carried out on intake structure was mainly focused on the optimization of pump-sump design [5-8], the analysis of the internal flow patterns and what measures shall be taken to reduce the swirl flow and vortex, and the effect comparison between different measures and their combinations [9-12], but few of them were related to the prediction of flow condition of pump and the calculation of hydraulic loss of sump at different intake water levels. There were plenty of numerical simulations on outflow structure; however, it was rare to study the performance of pumping system through calculation of hydraulic loss and combined with the pump performance.

Model tests of pumping system have direct and intuitive advantages, where attentions were chiefly paid to the observation of internal flow patterns, the checking of existence of flow separation, surface and subsurface vortices, what measures to be taken to improve the pumping system performance if necessary and validation of theoretical analysis and numerical simulation [13-16]. It is well known that the pump cavitation performance is usually tested on a closed test bench through reducing the atmospheric pressure of intake water surface. Hence, it was hardly possible to perform cavitation test of a pumping system with open surface intake design as the case to be studied, while the research funds were very limited and it was required that both the pump and the pumping system performance be tested in one model test. Reports were rare on model test of pumps with longer discharge pipes. The method introduced in the only one article that can be tracked was to divide the pumping system into two parts and carry out model tests for pump and discharge pipe, respectively [4], the disadvantage of which was higher cost and longer time.

The purpose of this paper is to realize the prediction of a mixed-flow pump and pumping system performance with the larger variation of intake water level and relatively longer discharge pipe. The methodology we applied to is to combine the technology of CFD and model test in terms of numerical simulation of sump and outflow structure and non-full passage model test of pumping system to save research funds and time.

\section{Research object and prediction method}

\subsection{Research object}

A certain core-pulling mixed-flow pump station was designed with open sump, where a total of six pump sets were installed, in which the impeller diameter is $1800 \mathrm{~mm}$, rotational speed is $333.3 \mathrm{r} / \mathrm{min}$, and the matched power of electric motor is $2500 \mathrm{~kW}$. The designed flow rate of the pump station is $56 \mathrm{~m}^{3} / \mathrm{s}$, and the intake is in the form of open sump design with water-suction bellmouth and round discharge pipe; the maximum, design and minimum head are $16.96 \mathrm{~m}, 13.59 \mathrm{~m}$ and $6.67 \mathrm{~m}$, respectively. In Fig. 1 is shown the sectional arrangement drawing of the pump station, which possesses such noticeable features as: (1) the variation of water level in the sump is quite large, and the biggest water depth difference in the sump is up to $9.12 \mathrm{~m}$; (2) the elevation difference from the bottom slab of sump to the motor floor extends to $20.40 \mathrm{~m}$; (3) the length from the entrance of sump to the outlet section of discharge pipe

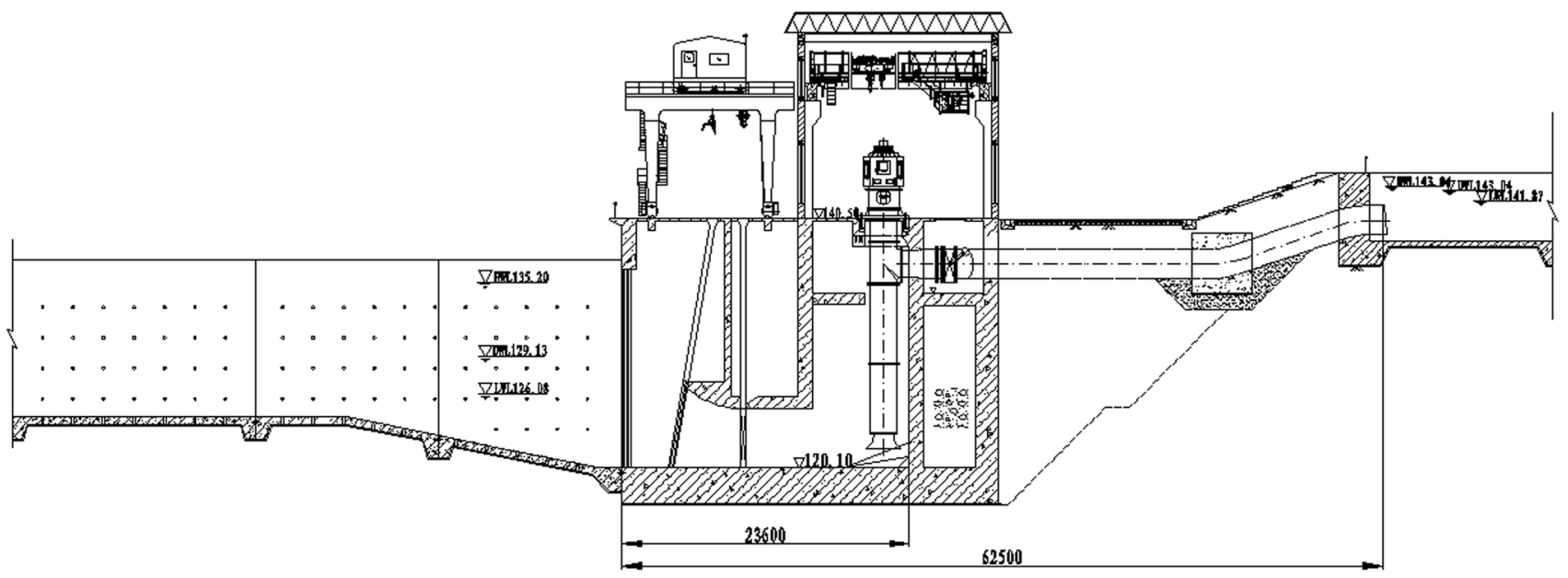

Fig. 1 The sectional arrangement drawing of the pump station. 1 Approaching channel; 2 forebay; 3 overhaul gate; 4 sump; 5 covering plate; 6 bellmouth; 7 outlet section of diffuser; 8 trash rack; 9 breast wall; 10 outlet of pump; 11 discharge pipe 
is $62.50 \mathrm{~m}$; (4) part of the sump is covered with submerged cover plate without free surface, and a breast wall is designed to reduce the height of overhaul gate and trash rack.

In the bidding document, it was stipulated that the hydraulic and cavitation performances of the prototype pump and pumping system be supplied by the pump manufacturer based on model test and all requirements be fully satisfied. There are several problems to be dealt with. (1) The cavitation performance cannot be carried out in an open test bench, while the free surface of sump cannot be simulated when the model test was conducted in a closed test bench. (2) Restricted by research funds and test periods, it is impossible to conduct performance test for pump and pumping system, respectively, so that the pump performance can only be extracted from a model pumping system test. (3) The impeller diameter of model pump has been set to be $364 \mathrm{~mm}$. If it is manufactured based on full passage simulation, the size of model pumping system will exceed the maximum limit of size of the test bench confirmed by the construction unit of the pumping station and the pump manufacturer. That means the height and length of the model pumping system should be decreased in some way; otherwise, the test cannot be performed. In considering those restriction factors, a method of combining numerical simulation with non-full passage physical model test of pumping system was proposed to realize the prediction of pump and pumping performance.

\subsection{Methodology for performance prediction of pump and pumping system}

In considering those restriction factors, a method of combining numerical simulation with non-full passage physical pumping system model test is proposed for prediction of the pump and pumping system performance.

The whole pumping system is divided into four parts, namely (1) the sump, (2) the part beginning from the watersuction bellmouth to the outlet section of pump diffuser, (3) the part beginning from the outlet section of pump diffuser to the outlet of pump and (4) the whole discharge pipe and part of discharge bay.

The following procedures shall be performed step by step to carry through numerical simulation and non-full passage model test and realize the performance prediction of the pump and pumping system.

(a) Numerical analysis is applied to calculate the hydraulic losses of sump, the segment from the outlet section of pump diffuser to the outlet section of pump and the whole discharge pipelines, respectively.

(b) The model test design for the non-full passage pumping system is performed and the manufacture of solid model followed. (c) The non-full passage pumping system model test is carried out, and the system performance parameters from the entrance of sump to the outlet section of pump diffuser are measured and calculated out.

(d) The model pump performance is figured out based on the hydraulic loss of sump and the one from the outlet section of pump diffuser to the pump outlet.

(e) Depending on the model pump performance, the model pumping system performance is figured out based on the hydraulic loss from the outlet section of pump diffuser to the outlet of discharge pipeline.

(f) The pump similarity law is applied to realize the performance conversion from the model pump and pumping system to the prototype ones.

\section{Prediction of hydraulic loss based on numerical analysis}

\subsection{Prediction of hydraulic loss of the sump}

\subsubsection{Structural features and three-dimensional modeling of sump}

In Fig. 2 is shown the plane figure of the sump, the structural design of which was different from that of a normal rectangle one, where several vortex-suppressing measures were taken, such as a transverse fillet between the back wall and the bottom slab, lateral contractions on each side wall and a bottom triangle pyramid just under the bottom of watersuction bellmouth [17-19].

The commercial modeling software Pro/E was adopted to establish the three-dimensional sump model for numerical simulation. The computation domain for the numerical simulation is shown in Fig. 3, including the sump, the watersuction bellmouth and an extension pipe.

\subsubsection{Meshes and setup for CFD analysis}

The commercial code Gambit was applied to generate unstructured four-face and structured six-face body meshes to accommodate complex structure of the computed domain. The total computation domain was divided into three parts, namely the front segment of sump, the rear segment of sump and the bellmouth and pipe. The mesh in the region of rear segment of sump, bellmouth and pipe was refined. The mesh numbers generated for the three parts were about 1.93 million, 2.40 million and 2.33 million, and the total number of meshes reached 6.66 million for the model sump at the designed intake water level, and the independence of mesh size was checked before the formal computation was commenced. 


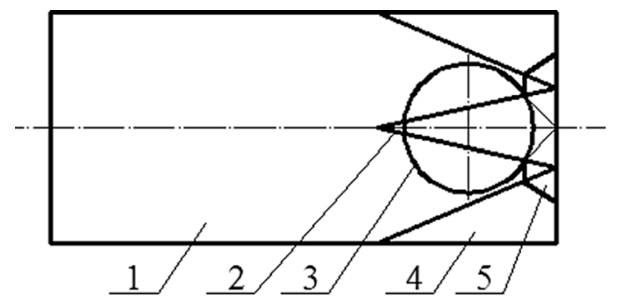

(a) The plane graph

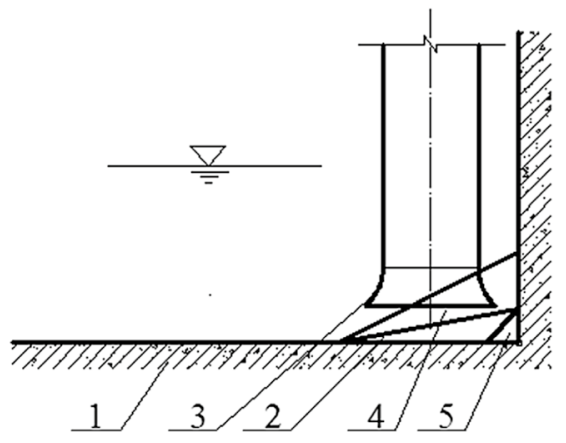

(b) The sectional drawing

Fig. 2 The structural feature of the sump. 1 Sump; 2 bottom triangle pyramid; 3 water-suction bellmouth; 4 lateral contraction; 5 transverse fillet

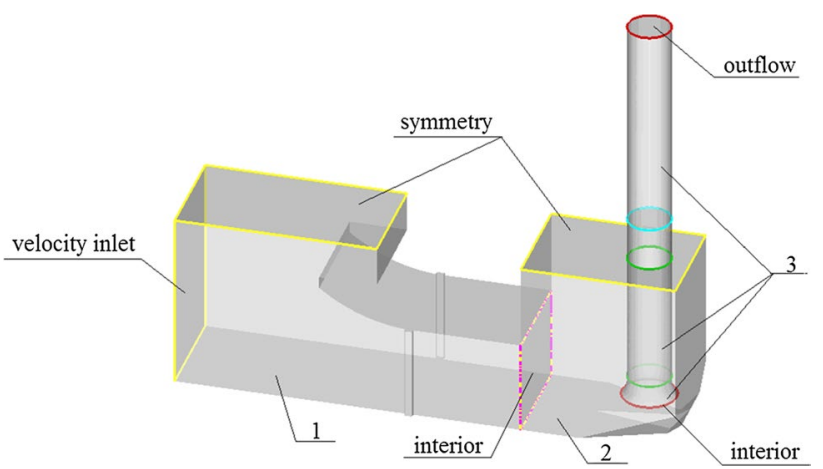

Fig. 3 The 3D modeling of sump and setup for CFD at the designed water level. 1 The front segment of sump; 2 the rear segment of sump; 3 the bellmouth and pipe

The commercial code FLUENT was applied to simulate the internal flow of sump. The governing equations for the turbulent incompressible flow were the steady-state RANS equations for the conservation of mass and momentum, and the $k-\varepsilon$ turbulence model was used to model the effective viscosity [20-23]. The inlet section of sump was defined as the velocity inlet, and the outlet section of the extension pipe was defined as outflow. The setup of boundary conditions is illustrated in Fig. 3. The interface between the front and the rear segment of sump was treated as interior and the same for the one between the rear segment of sump and the entrance section of bell mouth. The free water level was set as symmetry and all boundary surfaces of sump and pipe were treated as solid walls, satisfying no-slip conditions, and the standard wall functions were used to treat near-wall zone flows. The algorithm SIMPLEC was adopted to couple the calculation of velocity and pressure to improve computation efficiency and accelerate convergence [24-26].

\subsubsection{Analysis of internal flow of the sump}

In Fig. 4 are shown the velocity contours in the sump at different intake water levels and different flow rates, from which it can be seen that the flow pattern was smooth when water flowing into the water-suction bellmouth. Due to the vortex-suppressing effects of the transverse fillet, the lateral contractions and the bottom triangle pyramid, there were no phenomena of obvious collision and subsurface vortex. In the process of water flowing from the sump into the watersuction bellmouth, the flow fields on both sides of the sump were symmetry and the variation of velocity was basically even and steady.

According to the computation results, the velocity distribution uniformity in the suction pipe was greater than $93 \%$ and the maximum bias angle of inflow velocity was less than $1.0^{\circ}$ under different water levels. These technical indexes indicated that the intake design was successful and it can generate better flow conditions for pump, being beneficial to the full development of pump performance [27, 28].

\subsubsection{Calculation of hydraulic loss of the sump}

Based on simulation results, the hydraulic loss and drag coefficient of the sump at different water levels and different flow rates can be calculated out by applying the famous Bernoulli equation. From Table 1, it was seen that the hydraulic loss of sump varies with the flow rate and the intake water level. The higher the water level was, the greater the hydraulic loss would be. With the increase in the flow rate, the hydraulic loss increased accordingly. When the hydraulic loss is represented in terms of drag coefficient, its variation in value is relatively small, so that the hydraulic loss of sump can be expressed as the product of the drag coefficient multiplied by the square of flow rate. The arithmetic mean drag coefficient of the sump in different intake levels is equal to 0.465 .

\subsection{Prediction of hydraulic loss of discharge pipe based on numerical analysis}

The discharge pipe is composed of two parts. The first part starts from the outlet section of diffuser and ends at the outlet of pump. The second part is the rest pipeline, exclusive of 


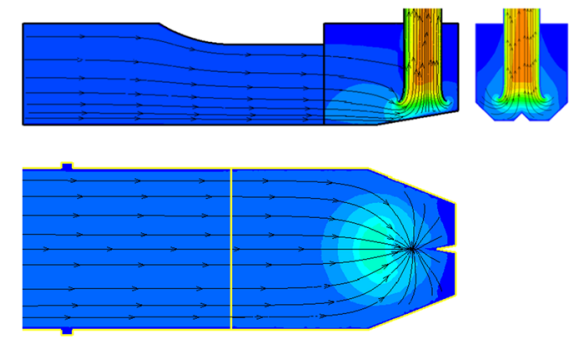

(a) At the lowest water level $\left(Q=0.400 \mathrm{~m}^{3} / \mathrm{s}\right)$
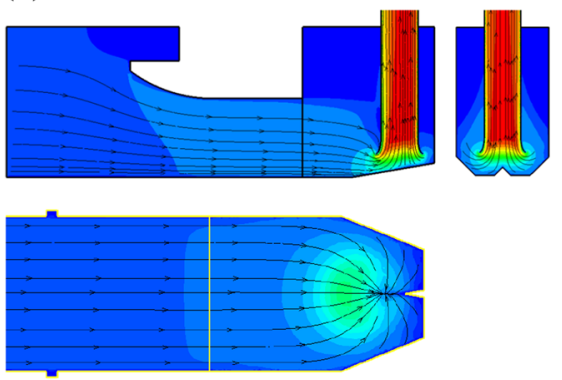

(b) At the design water level $\left(Q=0.458 \mathrm{~m}^{3} / \mathrm{s}\right)$
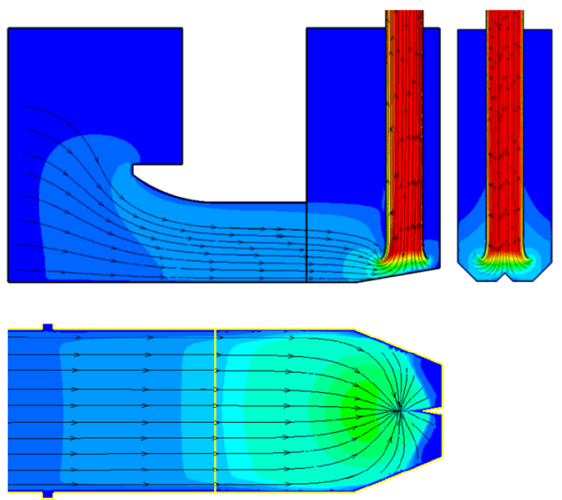

velocity-magnitude

$\begin{array}{lllllllllllll}0.25 & 0.53 & 0.81 & 1.09 & 1.37 & 1.65 & 1.93 & 2.21 & 2.49 & 2.77 & 3.05 & 3.33 & 3.61\end{array}$

(c) At the highest water level $\left(Q=0.531 \mathrm{~m}^{3} / \mathrm{s}\right)$

Fig. 4 The internal flow patterns of sump at different intake water levels

Table 1 The hydraulic loss and drag coefficient of the sump

\begin{tabular}{llll}
\hline Water level in sump & Flow rate $\left(\mathrm{m}^{3} / \mathrm{s}\right)$ & $\begin{array}{l}\text { Hydraulic } \\
\text { loss }(\mathrm{m})\end{array}$ & $\begin{array}{l}\text { Drag coeffi- } \\
\text { cient }\left(\mathrm{s}^{2} / \mathrm{m}^{5}\right)\end{array}$ \\
\hline Highest water level & 0.531 & 0.132 & 0.4681 \\
Design water level & 0.458 & 0.097 & 0.4629 \\
Lowest water level & 0.400 & 0.074 & 0.4625 \\
\hline
\end{tabular}

the first par, beginning from the outlet of pump. The numerical simulation domain is given in Fig. 5, inclusive of a pump barrel, a pump shaft, an outflow bent, a divergent pipe, two straight discharge pipe, two bents and a discharge bay. The method of setup for the numerical analysis of discharge pipe was similar with that used in the numerical simulation of sump, which were not repeated here. The outlet section of diffuser was defined as velocity inlet in the CFD analysis, and the outlet section of the discharge bay was defined as outflow of the computation domain. All interfaces were treated as interior. The free water surface of discharge bay was set as symmetry, and all boundary surfaces of pipe, bends and discharge bay were treated as solid walls.

Through numerical simulation, the internal flow patterns of discharge pipe at different flow rates can be obtained and the hydraulic losses can be calculated out [29, 30]. Given in Fig. 6 is the flow field in terms of velocity cloud when the flow rate was $0.531 \mathrm{~m}^{3} / \mathrm{s}$, from which it can be seen that there existed high-velocity zone and flow separation when water passed through the outflow bent and the divergent pipe. During the flowing toward the discharge bay along the discharge pipe, the velocity distribution became stable and even gradually, and when entered the discharge bay, the phenomena of submerged jets occurred due to the sudden enlargement of cross-sectional area.

Based on the computation results, the hydraulic loss of discharge pipe was directly proportional to the square of flow rate. When the flow rate was $0.531 \mathrm{~m}^{3} / \mathrm{s}$, the hydraulic loss from the outlet section of diffuser to the outlet of pump was $0.161 \mathrm{~m}$ and the corresponding drag coefficient was 0.634 , and the hydraulic loss and the corresponding drag coefficient for the discharge pipe were $0.513 \mathrm{~m}$ and 2.051, respectively.

\section{Model test design and layout of test bench}

\subsection{Model test design of non-full passage pumping system}

Aiming at the general arrangement and test requirements of the vertical pumping system, the design of model test for the non-full passage model pumping system was conducted while considering the testing capacity of a specific test bench [31]. In Fig. 7 is shown the specially designed non-full passage model pumping system.

Seen from Fig. 7, the following features were comprised in the design of model test: (a) the water surface was covered with plate, thus the open sump became an enforced intake, so that both of the model test of hydraulic and cavitation performance of pump can be carried out. (b) The length of pump barrel was shortened, and thus the total height of model pumping system can accommodate the allowed size of test bench. (c) The length of discharge pipe was shortened to decrease the total length of model pumping system. (d) The pressure-measuring section for calculation of the model pump head was set at the entrance section of the sump and the outlet section of the diffuser, 


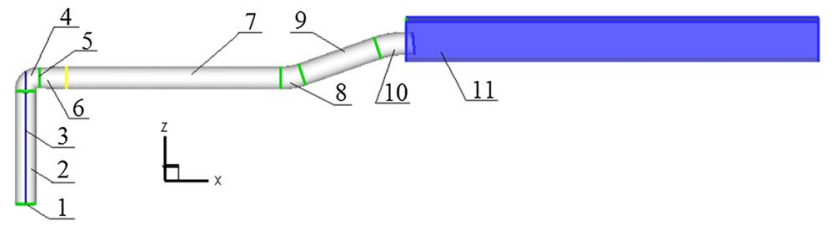

Fig. 5 The domain for computation of hydraulic loss of discharge pipe. 1 Outlet section of diffuser; 2 pump barrel; 3 pump shaft; 4 pump outlet bent; 5 outlet of pump; 6 divergent pipe; 7, 9 straight discharge pipe; 8,10 bent; 11 discharge bay

respectively. The measurement of flow rate, rotational speed and torque was the same as conventional model test of pumping system according to the relevant stipulations and standards [32, 33].

The non-full passage model pumping system was manufactured depending on the similarity law. In Fig. 8 is demonstrated a picture of the model pumping system installed on the test bench.

\subsection{Layout of test bench}

The test bench to be used is a closed circulating system with a water volume of $50 \mathrm{~m}^{3}$ (Fig. 9). The process of model test is conducted through a special program, and the test data are recorded automatically. Through switching of different shutoff valves, various hydraulic and cavitation performance test and runaway characteristic test of model pump and system can be carried out. The test bench has passed through technical appraisal by relevant departments of measurement quality supervision, the comprehensive error of which is $\pm 0.40 \%$.

In Table 2 are listed the technical indexes of main instruments and equipment used in the test bench for measurement of experimental parameters in the model test, and all instruments have been calibrated and working in the effective use period.

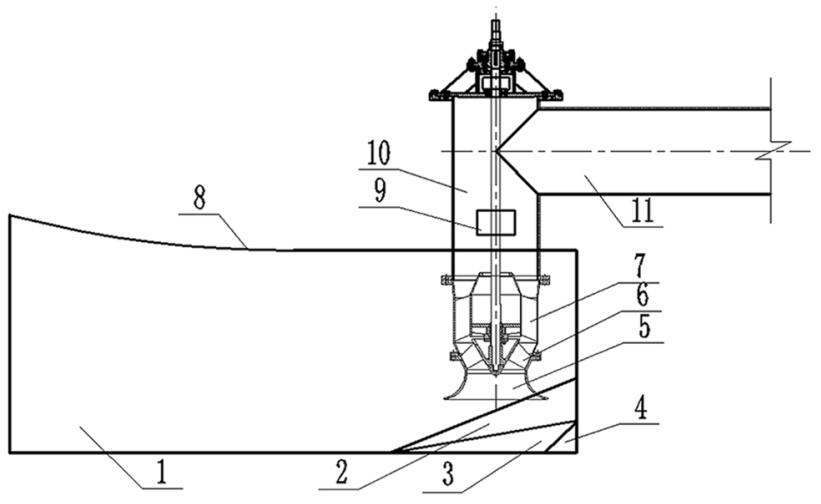

Fig. 7 The test design of non-full passage model pump system. 1 Sump; 2 lateral contraction; 3 bottom triangle pyramid; 4 transverse fillet; 5 water-suction bellmouth; 6 impeller; 7 diffuser; 8 covering plate; 9 observation window; 10 pump barrel; 11 discharge pipe

\section{Test results and analysis}

\subsection{Test results of non-full passage model pump}

\subsubsection{The hydraulic performance of non-full passage model pump}

As described previously, the impeller diameter and the rotational speed of the prototype pump are $1800 \mathrm{~mm}$ and $333.3 \mathrm{r} /$ min, respectively. Based on the similarity law of pump, it is known that the speed of the model pump shall be $1642.8 \mathrm{r}$ / min when the impeller diameter is $364 \mathrm{~mm}$.

The model test was carried out according to the relevant standards and criterions. It was found that the model pump device ran smoothly without harmful noise and vibration. The hydraulic performance of the non-full passage model pump is shown in Fig. 10. When the flow rate reached $504 \mathrm{~L} / \mathrm{s}$, the efficiency of the model pump was up to maximum of $88.46 \%$, and the corresponding pumping head was $13.51 \mathrm{~m}$.
Fig. 6 The velocity cloud of discharge pipe $(\mathrm{m} / \mathrm{s})$

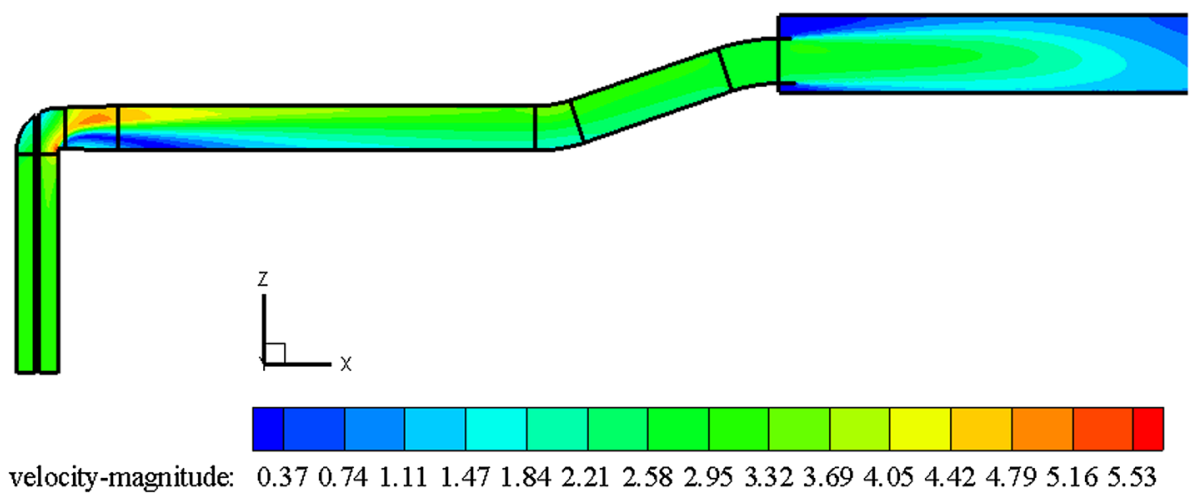




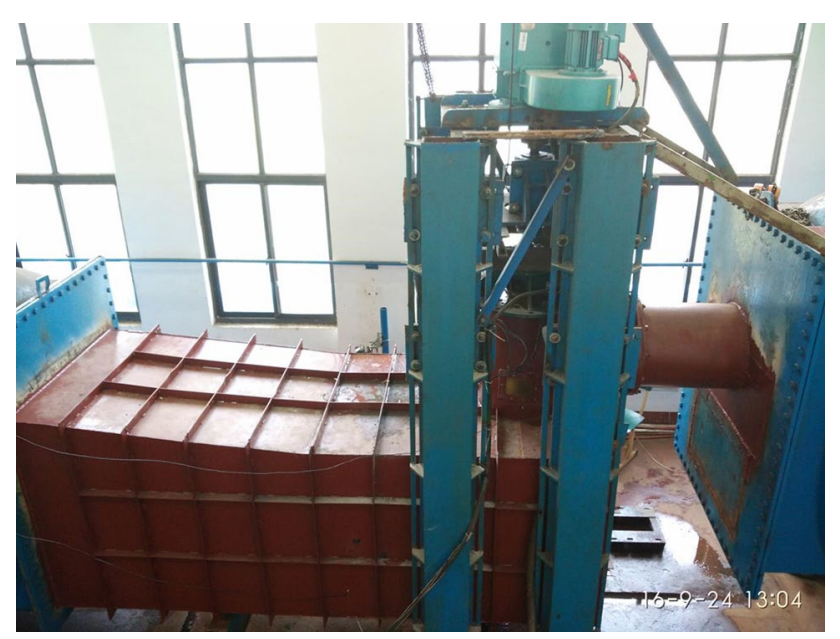

Fig. 8 Picture of the non-full passage model pumping system installed on test bench

\subsubsection{The cavitation performance of non-full passage model pump}

The cavitation characteristics are a vital performance index of pump, and the net positive suction head (NPSH) will affect directly the installation elevation of impeller and pump safety operation. The cavitation test was conducted through lowering the test system pressure by the vacuuming pump while keeping the flow rate unchanged. The critical NPSH was determined according to the dropping of pump efficiency by $1 \%$. In Fig. 11 is shown the cavitation performance of the model pump.

\subsection{Experimental uncertainties and error analysis}

There are errors in all test results. The uncertainties, or errors, in the model test of non-full passage pumping system were mainly caused by systematic errors and random errors $[34,35]$.

\subsubsection{Systematic uncertainty}

The efficiency was one of the main performance parameters of model pump, the test precision of which was directly related to the calibration accuracy of instruments used in the measurement of head, flow rate, torque and rotational speed of motor. To determine the uncertainty of test system in calculation of efficiency, the method of root-sum-square of each single systematic uncertainty was used, which is expressed by Eq. (1),

$E_{\eta s}= \pm \sqrt{E_{Q}^{2}+E_{H}^{2}+E_{T}^{2}+E_{n}^{2}}$

where $E_{\eta s}$ stands for the uncertainty of test system in calculation of pump efficiency; $E_{Q}$ is the uncertainty of test system in measurement of flow rate; $E_{H}$ stands for the uncertainty of test system in measurement of head; $E_{T}$ represents the uncertainty of test system in measurement of torque; and $E_{n}$ denotes the uncertainty of test system in measurement of rotational speed.

Hence, the uncertainty of test system in calculation of efficiency was easily figured out according to the calibration accuracy of different measurement instruments listed in Table 2.

$E_{\eta s}= \pm \sqrt{( \pm 0.10)^{2}+( \pm 0.20)^{2}+( \pm 0.20)^{2}+( \pm 0.10)^{2}}= \pm 0.316 \%$

\subsubsection{Random uncertainty}

The random uncertainty in the test process gave the repeatability estimation according to the discreteness of the efficiency value near the best efficiency point. According to the statistical principle, the formula for calculating random uncertainty is as follows.
Fig. 9 The schematic diagram of overall arrangement of the test bench. 1 Water inlet tank; 2 inlet water pressure-measuring section; 3 non-full passage model pumping system; 4 water outlet tank; 5 water tank; 6 electric gate valve; 7 electromagnetic flowmeter; 8 shutoff valve; 9 auxiliary pump; 10 DC speed regulated motor; 11 vacuum pump; 12 outlet water pressure-measuring section; 13 speed torque meter; 14 DC speed regulated motor

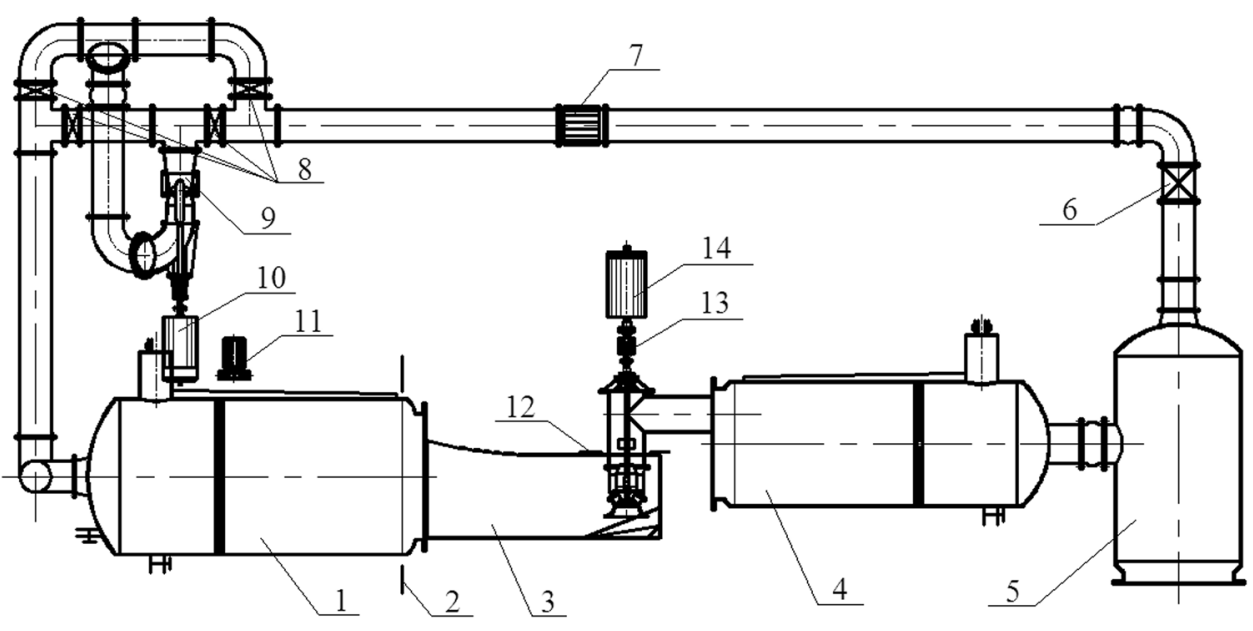


Table 2 List of main instruments and equipment used in the test bench

\begin{tabular}{|c|c|c|c|c|}
\hline Testing items & Name of instrument & Type & Scope of work & $\begin{array}{l}\text { Calibration } \\
\text { accuracy } \\
(\%)\end{array}$ \\
\hline Head & Differential pressure transmitter & EJA110A & $0-200 \mathrm{kPa}$ & \pm 0.10 \\
\hline Flow rate & Electromagnetic flowmeter & E-mag DN400 mm & $170-500 \mathrm{~L} / \mathrm{s}$ & \pm 0.20 \\
\hline \multirow[t]{2}{*}{ Rotational speed and torque } & Torque sensor & $\mathrm{JC} 1 \mathrm{~A}$ & $0-500 \mathrm{~N} \mathrm{~m}$ & \pm 0.20 \\
\hline & Digit display instrument & JW2A & 0-2000 rpm & \pm 0.10 \\
\hline Net positive suction head & Absolute pressure transmitter & EJA310A & $-100-130 \mathrm{kPa}$ & \pm 0.10 \\
\hline
\end{tabular}

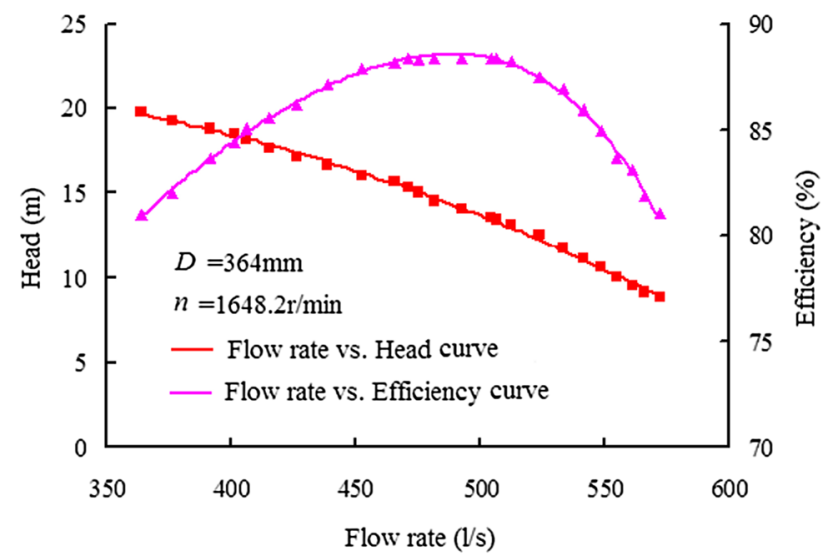

Fig. 10 The hydraulic performance of the non-full passage model pump

$E_{\eta r}=\frac{t_{0.95(n-1)} \times S_{\bar{\eta}}}{\bar{\eta} \sqrt{N}}$

In which $E_{\eta r}$ stands for the random uncertainty; $t_{0.95(n-1)}$ is the confidence coefficients corresponding to 0.95 and $(N-1)$ degrees of freedom; $N$ is the number of measurements. In this research, $N$ is equal to 20 , making $t_{0.95(n-1)}$ equal to $1.7291 ; \bar{\eta}$ denotes the arithmetic average efficiency, and here it is $88.47 \%$; $S_{\bar{\eta}}$ represents the standard deviation of the average efficiency, the value of which is equal to $0.128 \%$.

Based on the data given above, the random uncertainty in the calculation of efficiency is $\pm 0.113 \%$.

\subsubsection{Comprehensive uncertainty}

The comprehensive uncertainty $E_{\eta}$ in calculation of efficiency was the combination of systematic uncertainty and random uncertainty, and method of root-sum-square was adopted.

$$
\begin{aligned}
E_{\eta} & = \pm \sqrt{\left(E_{\eta s}\right)^{2}+\left(E_{\eta s}\right)^{2}} \\
& = \pm \sqrt{( \pm 0.316 \%)^{2}+( \pm 0.113 \%)^{2}}= \pm 0.336 \%
\end{aligned}
$$

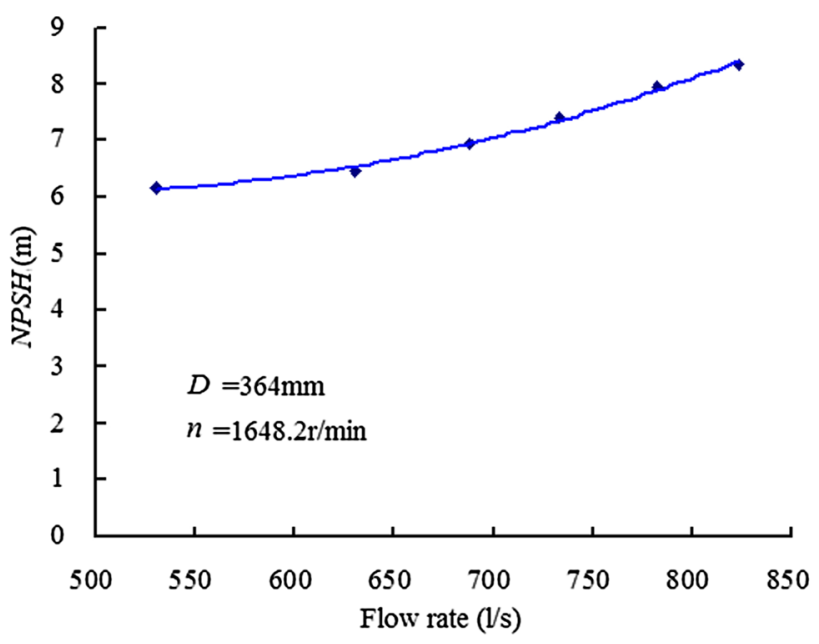

Fig. 11 The cavitation performance of the model pump

\section{Predictions of model pump and pumping system performance}

\subsection{Performance prediction of model pump}

For the hydraulic performance of the pump itself, the hydraulic loss of sump should not be included in its head; instead, the hydraulic loss from the outlet section of diffuser to the pump outlet was not included in the non-full passage model test. Therefore, the model test results need to be revised. On the basis of the non-full passage model test results and combined with the results of numerical analysis, the real pump performance can be obtained.

As we have known, the arithmetic mean drag coefficient of the sump in different intake levels was 0.465 and the drag coefficient from the outlet section of diffuser to the outlet of pump was 0.634 . Under the same flow rate, both the pump head and efficiency after revision will be lower than those obtained in model test. The larger the flow rate, the more obvious the decrease in head and efficiency was. The revised hydraulic performance of pump predicted through combination of model test and numerical analysis 
is shown in Fig. 12. The highest efficiency of model pump was $88.18 \%$. In comparison with that of non-full passage model pump and under the same flow rate, the highest efficiency of model pump decreased by $0.28 \%$ and the corresponding head dropped by $0.043 \mathrm{~m}$.

\subsection{Performance prediction of model pumping system}

The performance prediction of model pumping system was on the basis of model pump performance. Under the same flow rate, the difference in between the performance of model pump and model pumping system lay in the hydraulic loss produced in the sump and discharge pipe. Through the previous numerical simulation, this hydraulic loss varying with the flow rate has already been known, so that the pumping system performance can be calculated out. Given in Fig. 13 is the hydraulic performance of model pumping system. It can be seen from Fig. 13 that the highest efficiency of the model pumping system was $85.10 \%$, which was lower than that of the model pump under the same flow rate due to the counting of the hydraulic loss in sump and discharge pipe and the maximum decrease in efficiency was up to $7.60 \%$.

\section{Prediction of prototype pump performance}

\subsection{Prediction of hydraulic performance of prototype pump}

When the hydraulic performance of the model pump is known, the performance of the prototype pump can be converted by pump similarity law. There are several efficiency conversion formulas [36], and the one used in the case was given in the bidding document.

$$
\left\{\begin{array}{l}
Q_{P}=Q_{M}\left(\frac{n_{P}}{n_{M}}\right)\left(\frac{D_{P}}{D_{M}}\right)^{3} \\
H_{P}=H_{M}\left(\frac{n_{P}}{n_{M}}\right)^{2}\left(\frac{D_{P}}{D_{M}}\right)^{2} \\
N_{P}=N_{M}\left(\frac{n_{P}}{n_{M}}\right)^{3}\left(\frac{D_{P}}{D_{M}}\right)^{5} \\
\eta_{P}=1-\left(1-\eta_{M}\right)\left(0.3+0.7 \times \sqrt[5]{\frac{D_{M}}{D_{P}}} \sqrt[10]{\frac{H_{M}}{H_{P}}}\right)
\end{array}\right.
$$

where $D_{M}, n_{M}, Q_{M}, H_{M}, N_{M}$ and $\eta_{M}$ stand for the impeller diameter, rotational speed, flow rate, head, shaft power and efficiency of model pump, respectively. $D_{P}, n_{P}, Q_{P}, H_{P}, N_{P}$ and $\eta_{P}$ represent the impeller diameter, rotational speed, flow rate, head, shaft power and efficiency of prototype pump, respectively.

Given in Fig. 14 is the predicted hydraulic performance of prototype pump based on combination of non-full passage model test and numerical simulation and conversion formula. Compared with Fig. 12, it was known that the highest efficiency of prototype pump was $90.57 \%$ after the conversion between the model and prototype pump performance, which was $2.39 \%$ higher than that of the model pump, and the corresponding flow rate and head were $11.53 \mathrm{~m}^{3} / \mathrm{s}$ and $15.26 \mathrm{~m}$, respectively.

When the prototype pump ran under the maximum head $18.5 \mathrm{~m}$, the corresponding flow rate, efficiency and maximum shaft power were $9.81 \mathrm{~m}^{3} / \mathrm{s}, 87.34 \%$ and $2036 \mathrm{~kW}$, respectively. Since the pump was equipped with an electric motor of $2500 \mathrm{~kW}$, even if it operated under the maximum head, the matched power had more than $20 \%$ safety margin, meaning that the pump can run safely under extreme combination of water levels.

\subsection{Prediction of cavitation performance of prototype pump}

The conversion of cavitation performance between model and prototype pumps can be done by means of cavitation similarity law, which is expressed by the following formula.

$\operatorname{NPSH}_{P}=\operatorname{NPSH}_{M}\left(\frac{n_{P}}{n_{M}}\right)^{2}\left(\frac{D_{P}}{D_{M}}\right)^{2}$

In which $N P S H_{M}, n_{M}$ and $D_{M}$ denote the net positive suction head, rotational speed and impeller diameter of model pump, respectively. $\mathrm{NPSH}_{P}, n_{P}$ and $D_{P}$ represent the net positive suction head, rotational speed and impeller diameter of prototype pump, respectively.

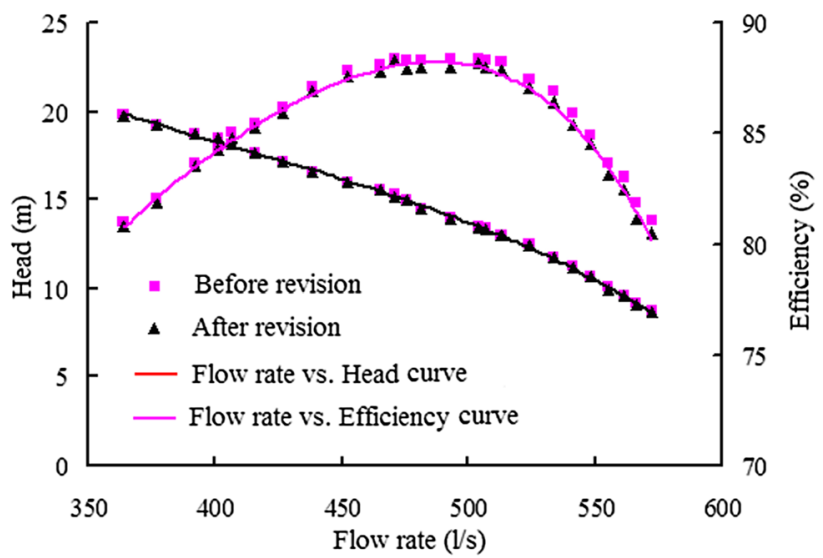

Fig. 12 The hydraulic performance of model pump 


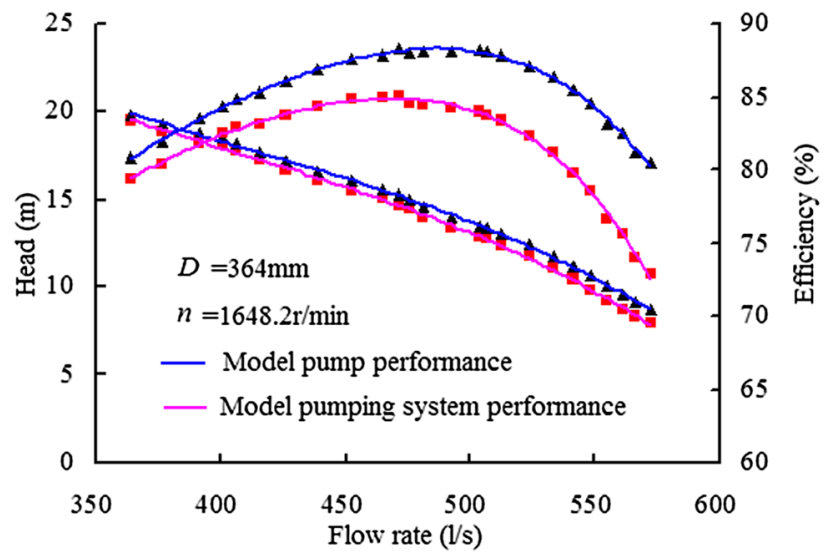

Fig. 13 The hydraulic performance of model pumping system

When designing the non-full passage model test, the method of $n_{M} D_{M}=n_{P} D_{P}$ was selected, so that the value of net positive suction head of model pump was equal to that of prototype pump while they ran under similar conditions. In Fig. 15 is the cavitation performance of prototype pump, with which the submersed depth of pump impeller can be calculated and the installing elevation of pump can be checked.

\subsection{Prediction of hydraulic performance of prototype pumping system}

The concerns of pump manufacturers and the pump users are not identical. The pump manufacturers focus on the design and manufacturing of their pump products, while the pump users pay more attentions to the pump selection and product quality and performance as well, and satisfying the design requirement is a thing of the first importance for designers and users of pump station. Whether the selected pump is suitable for the working conditions of pump station, the prototype pumping system performance will give the final answer.

Since the model and prototype pumping systems are similar, the hydraulic performances of them are inevitably similar when they are running under similar conditions in accordance with the similarity theory. Therefore, the prediction of prototype pumping system performance can be easily realized by applying the law of similarity, as shown in Fig. 16.

From Fig. 16, it can be seen that when the prototype pumping system was operated under the designed head of $13.59 \mathrm{~m}$, the corresponding flow rate and efficiency were $11.86 \mathrm{~m}^{3} / \mathrm{s}$ and $86.56 \%$, satisfying the requirements of $11.21 \mathrm{~m}^{3} / \mathrm{s}$ and $86.0 \%$, respectively, stipulated in the bidding document. Even if it ran under the maximum head of $16.96 \mathrm{~m}$, a flow rate of $10.30 \mathrm{~m}^{3} / \mathrm{s}$ could be achieved,

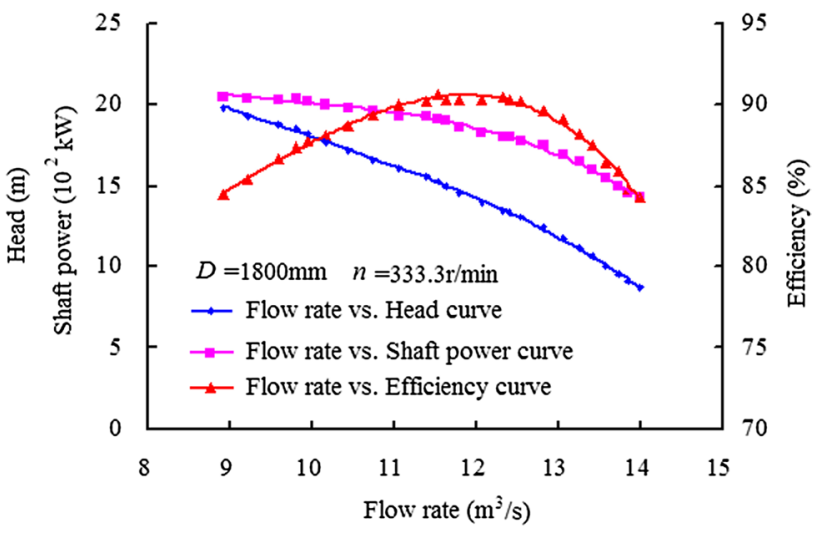

Fig. 14 The hydraulic performance of prototype pump

exceeding $9.33 \mathrm{~m}^{3} / \mathrm{s}$ required for each pump set, and the corresponding pumping efficiency was still as high as $86.11 \%$. The designed pumping capacity of $56 \mathrm{~m}^{3} / \mathrm{s}$ was fully satisfied. Hence, it can be concluded that the prototype pump possessed larger pumping capacity and excellent hydraulic performance.

\section{Conclusions}

1. Aiming at larger variation of water level in open sump and longer discharge pipe, and facing the requirements of pump and pumping performance prediction and restricted by the arrangement of test bench and shortage of funds, a new methodology was proposed to realize the performance prediction of pump and pumping system based on combination of numerical simulation and non-full passage model test.

2. The numerical simulation was carried out on sump and discharge pipe, respectively. The internal flow of sump with several vortex-suppressing measures was steady and smooth, and that water enters the water-suction bellmouth from all around without subsurface vortex, indicating that the intake design can generate better flow conditions for pump.

3. The calculation result showed that the impact of water level variation in sump was not obvious on its hydraulic loss and the drag coefficient is approximately a constant. The hydraulic loss in discharge pipelines was directly proportional to the square of flow rate.

4. The layout of the test bench and main measurement instruments were introduced. A non-full passage model test of pumping system was designed, and both the hydraulic and cavitation model tests were carried out. Detailed analysis of systematic uncertainty, random 


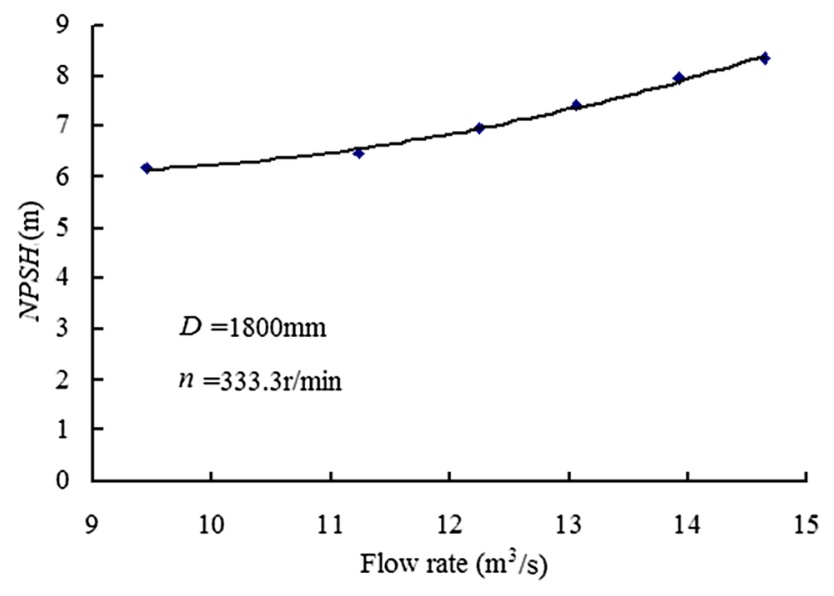

Fig. 15 The cavitation performance of prototype pump

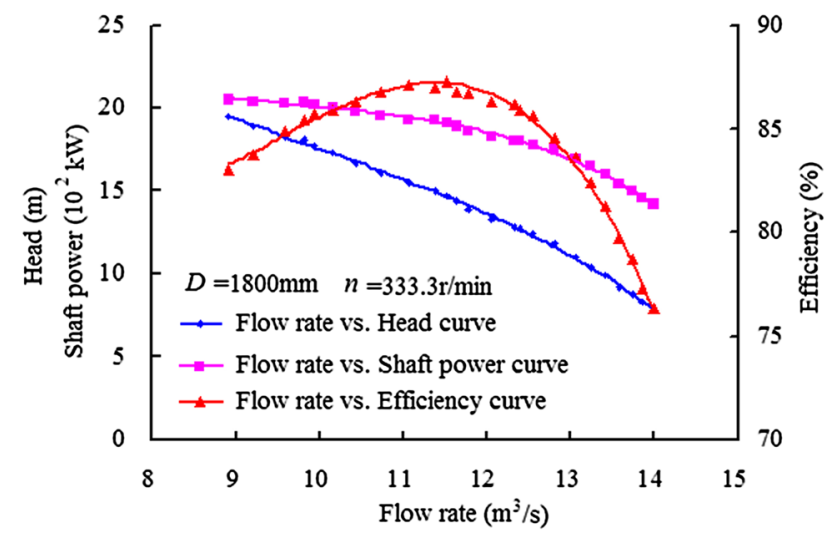

Fig. 16 The performance prediction of prototype pumping system

uncertainty and comprehensive uncertainty of the model test was conducted.

5. Based on the non-full passage model test of pumping system and combined with the numerical analysis of sump and discharge pipelines, the performance prediction of model pump and pumping system was realized after considering the hydraulic loss in the sump, in the flowing passage from the outlet section of diffuser to the outlet of pump and in the discharge pipe.

6. By applying the pump similarity law, the performances of prototype pump and pumping system were successfully predicted. The flow rate and efficiency of prototype pump were all up to the requirements of bidding documents. The prototype pumping system possessed larger pumping capacity and excellent hydraulic performance.

7. The methodology proposed in this paper highlights the flexibility of numerical analysis, and the advantages of intuitive and reliable of model test are fully brought into play, which can be referenced and applied to the engineering design of similar pump stations.

Acknowledgements This paper is financially supported by China National Science and Technology Supporting Program Grant (No. 2015BAB07B01) and China National Key Research and Development Projects: Integration and Application Demonstration of Water Control Technology in Artesian Irrigation Area (No. 2017YFC0403205).

Open Access This article is distributed under the terms of the Creative Commons Attribution 4.0 International License (http://creativeco mmons.org/licenses/by/4.0/), which permits unrestricted use, distribution, and reproduction in any medium, provided you give appropriate credit to the original author(s) and the source, provide a link to the Creative Commons license, and indicate if changes were made.

\section{References}

1. Liu C (2013) Pump and pump station. China Water \& Power Press, Beijing

2. Kan YG (2006) Controlling the installing quality of overhangwell-vertical-draw out core pumping set. Drain Irrigation Mach 24(5):20-24,28 (in Chinese)

3. Padmanabhan M, Hecker GE (1984) Scale effects in pump sump models. J Hydraul Eng ASCE 110(11):1540-1556

4. Chen S, Ge Q, Lu W et al (2003) The experimental method about pump setting characteristics with long discharge pipe. J Yangzhou Univ (Natural Science Edition) 6(4):78-82 (in Chinese)

5. Zhang J, Yee T (2018) Extent, capacity and possibilities of computational fluid dynamics as a design tool for pump intakes: a review. Water Sci Technol Water Supply 18(5):1518-1530

6. Ansar M, Nakato T, Constantinescu G (2002) Numerical simulations of inviscid three-dimensional flows at single and dual pump intakes. J Hydraul Res 40(4):461-470

7. Ahmad Z, Jain B, Kumar S (2011) Rational design of a pumpsump and its model testing. J Pipeline Syst Eng Pract 2(2):53-63

8. Teaima IR, El-Gamal T (2017) Improving flow performance of irrigation pump station intake. J Appl Water Eng Res 5(1):9-21

9. Choi JW, Choi YD, Kim CG et al (2010) Flow uniformity in a multi-intake pump sump model. J Mech Sci Technol 24(7):1389-1400

10. de Fockert A, van 't Westende JMC, Verhaart FIH (2015) Automatic swirl angle measurements for pump intake design. J Hydraul Res 53(3):384-393

11. Chuang WL, Hsiao SC (2011) Three-dimensional numerical simulation of intake model with cross flow. J Hydrodyn 23(3):314-324

12. Werth D, Frizzell C (2009) Minimum pump submergence to prevent surface vortex formation. J Hydraul Res 47(1):142-144

13. Rajendran VP, Constantinescu SG, Patel VC (1999) Experimental validation of numerical model of flow in pump-intake bays. $J$ Hydraul Eng ASCE 125(11):1119-1125

14. Kang WT, Shin BR, Doh DH (2014) An effective shape of floor splitter for reducing sub-surface vortices in pump sump. J Mech Sci Technol 28(1):175-182

15. Ansar M, Nakato T (2001) Experimental study of 3D pumpintake flows with and without cross flow. J Hydraul Eng ASCE 127(10):825-834

16. Bermudez M, Cea L, Puertas J et al (2017) Hydraulic model study of the intake-outlet of a pumped-storage hydropower plant. Eng Appl Comput Fluid Mech 11(1):483-495 
17. Fraser WH, Harrison NJ (1953) Hydraulic problems encountered in intake structures of vertical wet-pit pumps and methods leading to their solution. Am Soc Mech Eng 75:645-652

18. American National Standard for Pump Intake Design (1998) Pump intake design ANSI/HI 9.8. Hydraulic Institute of USA, Parsippansy, NJ, USA

19. US Army Corps of Engineers, Washington, DC (1988) Hydraulic design guidance for rectangular sumps of small pumping stations with vertical pumps and ponded approaches. Engineer Technology Letter No. 1110-2-313

20. Tao W (2001) Numerical heat transfer, 2nd edn. Xi-an Jiaotong University Press, China, Xi-an (in Chinese)

21. Yan C, Yu J, Xu J et al (2011) On the achievements and prospects for the methods of computational fluid dynamics. Adv Mech 41(5):562-589

22. Lu L, Zhang R (1997) Optimal hydraulic calculation of suction boxes in pump station. China Water \& Power Press, Beijing (in Chinese)

23. Zhang Q, Xu Y, Cao L et al (2017) A mixed-flow submersible well pump: design features and an investigation of performance. J Braz Soc Mech Sci Eng 39(7):2561-2569

24. Wang F (2005) Application of CFD to turbulent flow analysis and performance prediction in hydraulic machinery. J China Agric Univ 10(4):75-80 (in Chinese)

25. Costa Carosio GL, de Mendonca MT (2015) Lower pressure drop turbine blade trailing-edge cooling configuration. J Braz Soc Mech Sci Eng 37(4):1217-1233

26. Gao C, Xie K, Huang D et al (2015) Numerical simulation on the effect of the different water level on flow state of intake sump of pump station. J North China Univ Water Resour Electr Power (Natural Science Edition) 36(6):10-14 (in Chinese)

27. The Key Lab of Hydraulic and Power Engineering of Jiangsu Province (2016) Research report on the numerical analysis of sump and discharge pipelines for Longhai pump station, Yangzhou University (in Chinese)
28. Zhu H, Bin Xi (2003) New method for pump sump hydraulic model study. Trans Chin Soc Agric Mach 34(5):72-75 (in Chinese)

29. Zhu H, Yuan S, Liu H et al (2005) Numerical computation of the three-dimensional turbulent flow in siphon outlet conduit of large pumping stations. J Yangzhou Univ (Natural Science Edition) 8(2):74-78 (in Chinese)

30. Lu L, Liu J, Liang J et al (2008) Numerical simulation of 3D turbulent flow and hydraulic loss in outlet conduit of large pumping station. Drain Irrigation Mach 26(3):51-54 (in Chinese)

31. The Key Lab of Hydraulic and Power Engineering of Jiangsu Province (2016) Report on the model test of pump and pumping system for Longhai pump station. Yangzhou University (in Chinese)

32. The Standard of Ministry of Water Resources of PRC (2007) Code of practice for model pump and its installation acceptance tests (SL140-2006). China Water Power Press, China, Beijing

33. The Standard of People's Republic of China (2006) Rotodynamic pumps hydraulic performance acceptance tests: Grades 1 and 2 (GB/T 3216-2005). Standards Press of China, Beijing

34. Jia J, He X, Jin Y (2018) General theory of statistics. China Renmin University Press, China, Beijing

35. Li J (1987) Pump station testing technology. China WaterPower Press, China, Beijing

36. Zhu H, Yuan S, Liu H et al (2006) Research on conversion methods of hydraulic characteristics between prototype and model pumps and pumping systems. Trans Chin Soc Agric Mach 37(12):91-95 (in Chinese)

Publisher's Note Springer Nature remains neutral with regard to jurisdictional claims in published maps and institutional affiliations. 